\title{
Influence of the Storage Conditions (Frozen vs. Dried) in Health-Related Lipid Indexes and Antioxidants of Bee Pollen
}

\author{
Letícia M. Estevinho, Teresa Dias, and Ofélia Anjos*
}

Following harvest, bee pollen must be submitted to processing in order to maintain properties for consumers' health insurance. In this study, the changes on the lipid profile, contents of vitamin $C, \beta$-carotene and lycopene of bee pollen samples submitted to two conservation methods (freezing and drying) are evaluated. Eleven fatty acids, eight saturated, one monounsaturated, and two polyunsaturated are quantified. The PUFA/SFA ratio ranges from 1.18 to $3.95 \mathrm{~g} 100^{-1} \mathrm{~g}^{-1}$ and is significantly higher in the frozen extracts. On the other

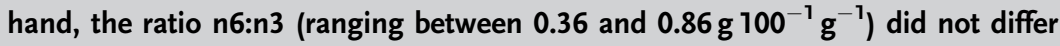
among processing methodologies, for most of the cases. The atherogenicity (AI) and thrombogenecity (TI) indexes are similar among preservation processes and coherent with the found on other health-promoting foods. The contents of vitamin $C, \beta$-carotene and lycopene are, for all samples, significantly superior in the frozen bee pollen.

Practical Applications: In the recent years the interest in natural products has mushroomed. Indeed, in addition to the good nutritional composition bee pollen possesses important bioactive compounds and promising health promoting activities that remain unstudied. In this study, it is evaluated the influence of the geographical origin and two storage methods (drying and freezing) in the fattyacid profile, lycopene, vitamin $C$ and $\beta$-carotene contents and on two lipid indexes of bee pollen. Results suggest that bee pollen may act as a complement for the prevention of atherosclerosis and thrombogenesis. Regarding storage it wi inferred that freezing allows preserving the bioactive compounds in a greater extent and, therefore, must be the preferred method. Further studies may be performed in order to take advantage of this natural products' potential.

\section{Introduction}

Bee pollen is produced by worker bees through the agglutination of flower pollen, nectar, and honeybee salivary substances. In the hive this product is packed in the honeycomb cells and covered with a layer of honey and wax generating bee bread, the principal source of bioactive nutrients and proteins for honey bee.

In general, bee pollen has a wonderful protein profile, providing appreciable amounts of essential amino acids such as methionine, lysine, threonine, histidine, leucine, isoleucine, valine, phenylalanine, and tryptophan $(10-40 \% \mathrm{w} / \mathrm{w})$. Also, it is a good source of carbohydrates $(13-55 \% \mathrm{w} / \mathrm{w})$, lipids $(1-13 \% \mathrm{w} / \mathrm{w})$, fiber $(0,3-20 \% \mathrm{w} / \mathrm{w})$ and biologically active compounds such as polyphenols $(2-5 \% \mathrm{w} / \mathrm{w})$, vitamins $(\beta$ Carotene, tocopherol, ascorbic acid, folic acid, biotin, complex B (B1- B6), and minerals (K, Mg, Ca, P, Fe, Zn, Cu, $\mathrm{Mn}) \cdot{ }^{[1,2]}$ As such, besides its importance for human nutrition, pollen contains several biological compounds such as flavonoids and other phenolic compounds that possess several pharmacological properties (antioxidant, antimicrobial, anti-inflammatory,

Prof. L. M. Estevinho, Prof. T. Dias

CIMO, Mountain Research Center

Polytechnic Institute of Bragança. Campus Santa Apolónia

5301-855 Bragança, Portugal

Prof. O. Anjos

Centro de Estudos Florestais

Instituto Superior de Agronomia

Universidade de Lisboa

1349-017 Lisboa, Portugal

E-mail: ofelia@ipcb.pt

Prof. O. Anjos

Instituto Politécnico de Castelo Branco

Quinta da Senhora de Mércules

6001-909 Castelo Branco, Portugal

Prof. O. Anjos

Centro de Biotecnologia de Plantas da Beira da Interior

1805 Castelo Branco, Portugal

The ORCID identification number(s) for the author(s) of this article can be found under https://doi.org/10.1002/ejlt.201800393.

DOI: 10.1002/ejlt.201800393 anti-mutagenic, hepatoprotective, and antiallergenic). ${ }^{[1-4]}$ Given these characteristics bee pollen could be used as a functional food and as dietary supplement with therapeutic effects.

The major determinant of variability in pollen chemical composition is the floral species, which can be affected by geographical region and climate conditions. ${ }^{[5]}$ This implies that each type of pollen has specific nutritional and nutraceutical characteristics. While analyses of the chemical composition are usually focused on the proteins and phenolic compounds available on bee pollen, its lipid profile has been unwell studied. Some authors analyzed the lipid profile of different bee pollen types $^{[6-8]}$ and found that the principal differences between the pollen samples were essentially related to the proportions of different fatty acids.

Additionally, another's changes in chemical composition of bee pollen can be introduced by beekeepers during the practices of cleaning, dehydration, packaging, and conservation applied to fresh pollen to increase the pollen shelf-life. ${ }^{[9]}$ Due to the high moisture level found in the composition of fresh pollen 
preservation processes are required to avoid the rapid fermentation and spoilage. ${ }^{[10]}$ Currently the commercial pollen is dehydrated at temperatures no higher than $40^{\circ} \mathrm{C}$ in a hot air chamber until the moisture reaches $7-10 \%$ without a standardized methodology. ${ }^{[2]}$ Despite the knowledge about the influence of drying process in nutritional and nutraceutical value of bee pollen is scarce, some studies showed a negative effect on the final content of $\beta$-carotene, vitamin C, E, pro-vitamin, ${ }^{[11,12]}$ polyphenols, ${ }^{[13]}$ free amino acids, ${ }^{[14]}$ and tocopherols.

In this background, the development of new approaches that allow reducing the moisture content of fresh pollen is crucial, promoting the preservation of nutritional and nutraceutical properties of bee pollen. In this context, the objective of this work was to evaluate the effect of two conservation methods (freezing and drying in greenhouse) on the lipid profile and vitamin $\mathrm{C}, \beta$ carotene, and lycopene after the drying treatment.

\section{Experimental Section}

\subsection{Bee Pollen Sampling and Preparation}

Nine bee pollen samples were harvested from beehives equipped with bottom-fitted pollen traps from three places in the Northeast of Portugal: Angueira (41 $\left.37^{\circ} 06^{\prime \prime} \mathrm{N} 6^{\circ} 24^{\prime} 22^{\prime \prime} \mathrm{O}\right)$, Vimioso ( $\left.41^{\circ} 34^{\prime} 57^{\prime \prime} \mathrm{N}, 6^{\circ} 31^{\prime} 59^{\prime \prime} \mathrm{W}\right)$ and Mogadouro $\left(41^{\circ} 20^{\prime} 0^{\prime \prime}\right.$ $\left.\mathrm{N}, 6^{\circ} 43^{\prime} 0^{\prime \prime} \mathrm{W}\right)$. The samples were collected in May of 2016 and the analyses were performed after 6 months of storage.

For quality and safety assurance, samples were studied regarding the presence of aerobic mesophiles, molds and yeasts, fecal coliforms, Escherichia coli, Clostridium spores, Bacillus cereus, Salmonella spp., and Staphylococcus aureus. For all samples, values were bellowing the hygienic standards recommended by Campos et al. ${ }^{[2]}$ The presence of aflatoxin B1, a widespread and dangerous mycotoxin, was excluded following the methodology described by Kostić et al. ${ }^{[8,15]}$ The botanical origin of the bee pollen samples was ascertained as previously described in detail by Feás et al. ${ }^{[16]}$ In brief, $2 \mathrm{~g}$ of each sample were grouped according to color and each subsample, corresponding to a botanical family, was then weighed to calculate its percentage in the main sample. Each subsample was then examined on an optical microscope with total magnification $(400 \times$ and $1000 \times)$. The reference collection of the Agricultural College of Bragança and different pollen morphology guides were used for pollen grains recognition.

Afterwards, each of the nine samples was divided into two aliquots: one was frozen at $-20^{\circ} \mathrm{C}$; while the other was thawed at room temperature and dried in an electric oven (ESA 1368, Sercon) with forced air circulation at $42{ }^{\circ} \mathrm{C}$, until reaching a moisture of $6-8 \%$. As such, for further analysis 18 samples of bee pollen, nine frozen and nine dried, were used.

\subsection{Determination of the Fatty-Acid Profile}

This determination was carried out as previously reported by Bárbara et al. ${ }^{[17]}$ First, crude fat was extracted with petroleum ether using an automatic Soxtec device (FOSS, Soxtec 2050, Höganäs, Sweden). Fatty acid methyl esters were then prepared from the extracted crude fat fraction by transesterification using $\mathrm{MeOH}$ in the presence of $\mathrm{H}_{2} \mathrm{SO}_{4}$. Then a portion containing $20 \pm 0.5 \mathrm{mg}$ of lipids was re-dissolved in $0.75 \mathrm{~mL}$ of $\mathrm{n}$-hexane and $0.1 \mathrm{~mL}$ of a solution of $2 \mathrm{~N} \mathrm{KOH}$ in $\mathrm{MeOH}$ was added. The solution was mixed in a vortex mixer (Model Reax 2000, Schwabach, Germany) for $2 \mathrm{~min}$ and then dried over anhydrous $\mathrm{Na}_{2} \mathrm{SO}_{4}$. Following phase separation, a portion from the superior organic layer (containing fatty acids and an internal standard) was injected into the fatty acid determination system (B-820). The fatty acids were separated by gas chromatography and were detected using flame ionization. The fatty-acid methyl esters were identified by comparing the retention times of the peaks obtained in the bee pollen sample with those of known reference esters.

The results of three independent replicates were recorded and processed using software Clarity SW 1.7 (DataApex, Podohradska, Czech Republic). This software automatically calculated the contents of fat, saturated, mono-unsaturated fatty acids (MUFA), poly-unsaturated fatty acids (PUFA) as well as the content of each individual fatty acidy (expressed as g per $100 \mathrm{~g}$ of bee pollen sample). The atherogenic (AI) and the thrombogenic (TI) indexes were calculated according to the equations provided in Ghaeni et al. ${ }^{[18]}$

\section{3. $\beta$-Carotene Content Determination}

Following the methodology reported by Montenegro et al., ${ }^{[19]} 1 \mathrm{~g}$ of each bee pollen sample was weighed into a beaker and macerated with $10 \mathrm{~mL}$ mixture of acetone and n-hexane (1:1). After filtration, $10 \mathrm{~mL}$ of a solution of $\left(\mathrm{NH}_{4}\right)_{2} \mathrm{SO}_{4}$ at $50 \%$ were added and vigorously shaken. Then, the superior layer was collected and the absorbance read (espectrofotometer). In order to minimize the lycopene interference in the calculation of $\beta$ carotene concentration, a calibration plane relating the concentrations from standards (Sigma Chemical, St. Louis, USA) and absorbances at $452 \mathrm{~nm}$ (positive correlation) and $510 \mathrm{~nm}$ (negative correlation), as described by Adalid et al. ${ }^{[20]}$ it was calculated. Each measurement was performed in triplicate and results are expressed as $\mu \mathrm{gg}^{-1}$ of bee pollen sample.

\subsection{Lycopene Content Determination}

The determination of lycopene was performed as described by Montenegro et al. ${ }^{[19]}$ by adding a mixture of hexane/ethanol/ acetone $(2: 1: 1 \mathrm{v} / \mathrm{v} / \mathrm{v})$ containing $0.05 \%$ of BHT to $1 \mathrm{~g}$ of each bee pollen sample. In order to prevent lycopene photo-oxidation and isomerization, pollen maceration was performed under dimmed light in glassware wrapped by aluminum foil. Calibration curves of lycopene standard $\left(R^{2}=0.99\right)$ were used for lycopene content determination and absorbance was read at $540 \mathrm{~nm}$ in espectrofotometer. Each measurement was performed in triplicate and results are expressed as $\mu \mathrm{gg}^{-1}$ of bee pollen sample.

\subsection{Determination of the Vitamin C content}

This determination was performed as recommended by $\mathrm{AOAC}^{[21]}$ and Oliveira et al. ${ }^{[22]}$ One gram of each bee pollen 
sample was weighed and extracted twice with an aqueous solution of metaphosphoric acid-acetic acid at $14.000 \mathrm{rpm}$ for $10 \mathrm{~min}$. After this, the supernatant was transferred to a flask of $50 \mathrm{~mL}$ and the volume was completed with the acid. This solution was potentiometric titrated with 2,6-dichlorophenolindophenol until end point of the titration. A standard solution was prepared and titrated. The concentration of vitamin $\mathrm{C}$ was ascertained using the following equation, where $\mathrm{SC}=$ standard concentration; $\mathrm{V}=$ volume used for titration; $\mathrm{w}=$ dry-weight basis; V=volume used in standard solution titration. Each measurement was performed in triplicate and results are expressed as $\mu \mathrm{gg}^{-1}$ of bee pollen sample.

$\mu \mathrm{g}$ vitamin $\mathrm{C} / \mathrm{g}$ bee pollen $=\frac{\mathrm{SC} \times \mathrm{V} \times 100}{\mathrm{w} \times \mathrm{v}}$

\subsection{Statistical Analysis}

A two-way analysis of variance (ANOVA) was performed to analyses the effects of the different samples with different predominate pollen, codified as species (nine levels); and the storage method (two levels: Frozen (Fz) and Dehydrate (Dh)) as fixed factors. The percentage of variance of each factor as well for interaction between factor and residual were calculated based on the mean square values obtain in the ANOVA test. Following ANOVA, means comparison was performed with a LSD post hoc test with $95 \%$ confidence intervals. In the results an equal letter represents that the values obtained do not differ statistically $(p>0.05)$.

The results were also subjected to a multivariate analysis (principal component analysis) to study the association between variables and cases.

The samples were codified according the predominant botanical family of pollen grains: sample $1-$ Rubus spp. (Ru); sample 2 - Castanea sativa (Ca); sample 3 - Quercus spp (Qu); sample 4 - Leontondon spp. (Le); sample 5 - Cytisus spp.
(Cy); sample 6 - Echium spp. (Ec); sample 7 - Castanea sativa and Cistus spp. (Ca and Ci); sample 8 - Eucalyptus spp. and Erica spp. (Eu and Er); sample 9 - Erica spp. (Er).

The experimental data were analyzed using statistic Statistics version 7.0.

\section{Results}

\subsection{Pollen Analysis}

From the palynological analysis (Table 1) seven of the samples could be classified as monofloral since those presented more than $90 \%$ of the pollen grains belonging to a particular botanical type. The remaining two were heterofloral, with predominance of i) Castanea sativa and Echium spp. and ii) Erica spp. and Eucalyptus spp.

\subsection{Lipid Fraction Characterization}

The fatty acids (FA) profile, comprising saturated FA (SFA), monounsaturated FA (MUFAs), and polyunsaturated FA (PUFAs) of the frozen and dried apicultural pollen samples are presented in Tables 2-4.

In general, the preservation process did not interfere on PUFAs concentration $(p>0.05)$. However, the amount of SFA and MUFA differed depending on the preservation method being the fatty acids' concentrations superior in the dried bee pollen samples.

Eleven fatty acids, eight saturated, one monounsaturated and two polyunsaturated were identified and quantified. The values obtained for SFA ranged between $0.69 \pm 0.07$ and $1.80 \pm 0.12$ $\mathrm{g} 100^{-1} \mathrm{~g}^{-1}$; the results obtained for MUFAs were between

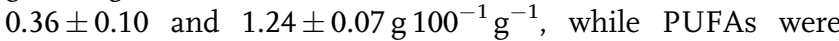

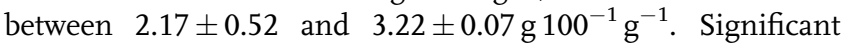
differences were not observed on the fatty acid profile of the analyzed samples (Table 2).

Table 1. Sample code and pollen spectrum of different bee pollen samples used in the study.

\begin{tabular}{|c|c|c|c|c|c|c|c|c|c|}
\hline \multirow[b]{2}{*}{ Pollen type } & \multicolumn{9}{|c|}{ Samples } \\
\hline & $\mathrm{Ru}$ & $\mathrm{Ca}$ & $\mathrm{Ci}$ & Le & Cy & $\mathrm{Ec}$ & $\mathrm{Ca}+\mathrm{Ec}$ & $\mathrm{Eu}+\mathrm{Er}$ & $\mathrm{Er}$ \\
\hline Rubus spp. & $89.6 \pm 2.8$ & & $2.0 \pm 0.6$ & & $1.1 \pm 0.6$ & & $3.6 \pm 1.0$ & & \\
\hline Castanea sativa & $2.5 \pm 0.5$ & $91.0 \pm 3.2$ & $2.2 \pm 0.7$ & $2.5 \pm 1.7$ & $2.0 \pm 0.7$ & $2.3 \pm 0.9$ & $37.2 \pm 2.5$ & & \\
\hline Cytisus spp. & $<1$ & $2.0 \pm 0.7$ & & $1.6 \pm 1.6$ & $90.0 \pm 3.1$ & & & & $3.2 \pm 1.9$ \\
\hline Quercus spp. & $<1$ & $2.0 \pm 0.8$ & & $<1$ & & $2.5 \pm 0.5$ & & $12.3 \pm 0.8$ & \\
\hline Echium spp. & $2.2 \pm 0.8$ & & $2.6 \pm 0.6$ & $1.5 \pm 0.6$ & $2.7 \pm 0.9$ & $89.8 \pm 2.0$ & $23.4 \pm 3.4$ & $7.7 \pm 2.2$ & \\
\hline Prunus spp. & & $2.3 \pm 1.2$ & & $1.1 \pm 0.6$ & & & & $11.5 \pm 2.5$ & $<1$ \\
\hline Leontondon spp. & $2.0 \pm 0.8$ & & $1.9 \pm 0.5$ & $85.6 \pm 4.3$ & $1.2 \pm 0.5$ & & $12.6 \pm 1.7$ & & $1.5 \pm 0.6$ \\
\hline Eucalyptus spp. & & $2.7 \pm 1.1$ & $3.0 \pm 0.3$ & $<1$ & & & & $27.4 \pm 1.8$ & \\
\hline Erica spp. & $2.5 \pm 0.8$ & & $<1$ & $1.9 \pm 0.5$ & $1.5 \pm 1.0$ & & $8.1 \pm 1.0$ & $27.6 \pm 2.4$ & $89.9 \pm 4.2$ \\
\hline Cistus spp. & & & $87.7 \pm 2.4$ & $2.5 \pm 1.0$ & $1.5 \pm 0.4$ & $5.3 \pm 1.1$ & $15.1 \pm 2.2$ & & $2.0 \pm 0.7$ \\
\hline Trifolium spp. & & & & $2.2 \pm 1.6$ & & & & $13.5 \pm 1.1$ & $3.0 \pm 1.2$ \\
\hline
\end{tabular}

Ru, Rubus spp.; Ca, Castaneia sativa; Qu, Quercus spp.; Le, Leontondon spp.; Cy, Cytisus spp.; Ec, Echium spp.; Ci, Cistus spp.; Eu, Eucalyptus spp.; Er, Erica spp. 
Table 2. Saturated, monounsaturated, and polyunsaturated fatty acids of the frozen and dried apicultural pollen samples.

\begin{tabular}{|c|c|c|c|c|c|c|}
\hline Sample & Code & $\operatorname{TF}\left(\mathrm{g} 100^{-1} \mathrm{~g}^{-1}\right)$ & SFA $\left(g 100^{-1} g^{-1}\right)$ & MUFA $\left(\mathrm{g} 100^{-1} \mathrm{~g}^{-1}\right)$ & 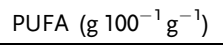 & N.I. $\left(\mathrm{g} 100^{-1} \mathrm{~g}^{-1}\right)$ \\
\hline \multirow[t]{2}{*}{$\mathrm{Ru}$} & $\mathrm{F}$ & $3.74 \pm 0.54^{\mathrm{a}}$ & $0.72 \pm 0.10^{\mathrm{a}}$ & $0.44 \pm 0.06^{\mathrm{a}}$ & $2.41 \pm 0.38^{\mathrm{a}}$ & $0.19 \pm 0.12^{\mathrm{a}}$ \\
\hline & $\mathrm{D}$ & $4.39 \pm 0.07^{\mathrm{a}}$ & $1.17 \pm 0.18^{b}$ & $0.51 \pm 0.07^{\mathrm{a}}$ & $2.48 \pm 0.17^{\mathrm{a}}$ & $0.24 \pm 0.05^{b}$ \\
\hline \multirow[t]{2}{*}{$\mathrm{Ca}$} & $\mathrm{F}$ & $3.56 \pm 0.78^{\mathrm{a}}$ & $0.78 \pm 0.20^{\mathrm{a}}$ & $0.40 \pm 0.08^{\mathrm{a}}$ & $2.17 \pm 0.52^{\mathrm{a}}$ & $0.22 \pm 0.06^{\mathrm{a}}$ \\
\hline & $\mathrm{D}$ & $4.55 \pm 0.29^{\mathrm{a}}$ & $1.08 \pm 0.17^{\mathrm{a}}$ & $0.65 \pm 0.09^{b}$ & $2.57 \pm 0.16^{\mathrm{a}}$ & $0.25 \pm 0.05^{\mathrm{a}}$ \\
\hline \multirow[t]{2}{*}{ Qu } & $\mathrm{F}$ & $4.00 \pm 0.31^{\mathrm{a}}$ & $0.88 \pm 0.15^{\mathrm{a}}$ & $0.65 \pm 0.10^{\mathrm{a}}$ & $2.28 \pm 0.31^{\mathrm{a}}$ & $0.19 \pm 0.03^{\mathrm{a}}$ \\
\hline & D & $5.15 \pm 0.45^{\mathrm{a}}$ & $1.19 \pm 0.09^{\mathrm{a}}$ & $0.95 \pm 0.07^{b}$ & $2.77 \pm 0.25^{\mathrm{a}}$ & $0.25 \pm 0.07^{b}$ \\
\hline \multirow[t]{2}{*}{ Le } & $\mathrm{F}$ & $4.94 \pm 0.33^{\mathrm{a}}$ & $1.16 \pm 0.23^{\mathrm{a}}$ & $0.93 \pm 0.17^{\mathrm{a}}$ & $2.65 \pm 0.09^{\mathrm{a}}$ & $0.20 \pm 0.04^{\mathrm{a}}$ \\
\hline & D & $6.40 \pm 0.39^{\mathrm{b}}$ & $1.80 \pm 0.12^{\mathrm{b}}$ & $1.16 \pm 0.14^{\mathrm{a}}$ & $3.22 \pm 0.07^{\mathrm{b}}$ & $0.22 \pm 0.09^{\mathrm{a}}$ \\
\hline \multirow[t]{2}{*}{ Cy } & $\mathrm{F}$ & $3.96 \pm 0.64^{\mathrm{a}}$ & $0.96 \pm 0.22^{\mathrm{a}}$ & $0.67 \pm 0.06^{\mathrm{a}}$ & $2.19 \pm 0.36^{\mathrm{a}}$ & $0.15 \pm 0.06^{\mathrm{a}}$ \\
\hline & D & $5.27 \pm 0.90^{\mathrm{a}}$ & $1.65 \pm 0.26^{\mathrm{a}}$ & $1.24 \pm 0.07^{\mathrm{a}}$ & $2.20 \pm 0.53^{\mathrm{a}}$ & $0.17 \pm 0.05^{\mathrm{a}}$ \\
\hline \multirow[t]{2}{*}{ Ec } & $\mathrm{F}$ & $4.06 \pm 0.35^{\mathrm{a}}$ & $0.83 \pm 0.09^{\mathrm{a}}$ & $0.46 \pm 0.18^{a}$ & $2.66 \pm 0.21^{\mathrm{a}}$ & $0.12 \pm 0.01^{\mathrm{a}}$ \\
\hline & D & $5.86 \pm 0.55^{\mathrm{a}}$ & $1.54 \pm 0.27^{b}$ & $0.84 \pm 0.17^{\mathrm{a}}$ & $3.20 \pm 0.23^{b}$ & $0.29 \pm 0.04^{b}$ \\
\hline \multirow[t]{2}{*}{$\mathrm{Ca}$ and $\mathrm{Ci}$} & $\mathrm{F}$ & $4.52 \pm 0.25^{\mathrm{a}}$ & $1.18 \pm 0.09^{\mathrm{a}}$ & $0.62 \pm 0.12^{\mathrm{a}}$ & $2.55 \pm 0.16^{\mathrm{a}}$ & $0.17 \pm 0.06^{\mathrm{a}}$ \\
\hline & D & $5.64 \pm 0.44^{\mathrm{a}}$ & $1.41 \pm 0.21^{\mathrm{a}}$ & $0.98 \pm 0.13^{b}$ & $3.00 \pm 0.28^{\mathrm{a}}$ & $0.25 \pm 0.05^{\mathrm{a}}$ \\
\hline \multirow[t]{2}{*}{$\mathrm{Eu}$ and $\mathrm{Er}$} & $\mathrm{F}$ & $4.70 \pm 0.04^{\mathrm{a}}$ & $1.00 \pm 0.10^{\mathrm{a}}$ & $0.65 \pm 0.08^{\mathrm{a}}$ & $2.87 \pm 0.08^{\mathrm{a}}$ & $0.18 \pm 0.04^{\mathrm{a}}$ \\
\hline & D & $5.70 \pm 0.42^{b}$ & $1.61 \pm 0.08^{b}$ & $0.91 \pm 0.14^{b}$ & $2.98 \pm 0.19^{\mathrm{a}}$ & $0.21 \pm 0.05^{\mathrm{a}}$ \\
\hline \multirow[t]{2}{*}{$\mathrm{Er}$} & $\mathrm{F}$ & $3.41 \pm 0.38^{\mathrm{a}}$ & $0.69 \pm 0.07^{\mathrm{a}}$ & $0.36 \pm 0.10^{\mathrm{a}}$ & $2.24 \pm 0.37^{\mathrm{a}}$ & $0.13 \pm 0.01^{a}$ \\
\hline & D & $5.30 \pm 0.71^{b}$ & $1.30 \pm 0.12^{b}$ & $0.96 \pm 0.04^{b}$ & $2.79 \pm 0.61^{\mathrm{a}}$ & $0.25 \pm 0.10^{b}$ \\
\hline
\end{tabular}

Ru, Rubus spp.; Ca, Castaneia sativa; Qu, Quercus spp.; Le, Leontondon spp.; Cy, Cytisus spp.; Ec, Echium spp.; Ci, Cistus spp.; Eu, Eucalyptus spp.; Er, Erica spp. TF, total fat; SFA, saturated fatty acids; MUFA, monounsaturated fatty acids; PUFA, poly unsaturated fatty acids; N.I., non-identified fatty acids.

In general, the preservation process did not interfere on PUFAs concentration $(p>0.05)$. However, the amount of SFA and MUFA differed depending on the preservation method. Even though the FA concentrations differed depending on the conservation processes, the samples Echium spp. and Leontondon spp. possessed, in all cases, a greater amount of PUFA (Table 2).

In the present study, the predominance of $\alpha$-linolenic acid (C18:3n3) (ranging from $2.15 \pm 0.09$ to $1.27 \pm 0.24 \mathrm{~g} \mathrm{100}^{-1} \mathrm{~g}^{-1}$ was observed in all samples. Indeed, these concentrations were approximately twofold higher than those of linoleic acid (ranging

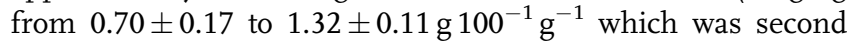
more prevalent (Table 4)).

The third most abundant fatty acid was palmitic (C16:0), followed by oleic acid (C18:1n9). Significantly higher levels of (C16:0) were obtained in the frozen extracts of Ru, Ca, Le, Ec, Eu, and Er compared to those obtained in dehydrated extracts. On the other hand, regarding the oleic acid, statistical differences were not found between frozen and dehydrated samples $\mathrm{Ru}, \mathrm{Ci}$, Le, $\mathrm{Cy}, \mathrm{Eu}$, and Er.

The fatty acids C4:0, C6:0, C8:0, C10:0, C12:0, C14:0, and $\mathrm{C} 18: 0$ were also quantified in the dried and frozen bee pollen samples yet in much lower concentrations (Tables 3 and 4).

Given the variance analysis results presented in Table 5, it is possible observed that for almost all parameters the species and the storage methods are highly significant $(p<0.001)$. Only for N.I. [non-identified fatty acids] and C18:2n6c the effect of the species are not significant to explain the differences between samples.

The fatty acids that present a higher difference between samples were the C:10, C:12, and C18:3n3, that explain respectively $43.6,42.7$, and $41.5 \%$ of the total variance.
Concerning the storage method, the ANOVA results clearly show the higher difference between both methods with a percentage of variance ranging between $12.7 \%$ for $\mathrm{C} 18: 3 \mathrm{n} 3$ and 61.5\% for C16:0. This influence is also presented in Figure 1 that represent the projection of the pollen samples on the plane of the two main factors resulting from principal component analysis (PCA) for the individual fatty acid. The PCA explain $74.5 \%$ of the total variance observed.

Regarding the variability observed in the pollen samples (given by the residual column in ANOVA Table) the values are higher. In fact, each pollen sample have a predominant pollen, but also a minor percentage of other pollens that explain this variability in their properties.

The PUFA/SFA ratio ranged from $1.32 \pm 0.12$ to $3.37 \pm$

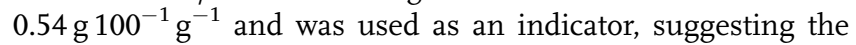
nutritional quality of the lipid fraction of food. In general it was significantly higher in frozen extracts, particularly in Ec and Ru samples (Table 6).

Regarding the ratio $\mathrm{n} 6: \mathrm{n} 3$ (ranging from $0.41 \pm 0.06$ to

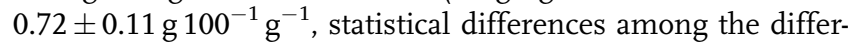
ent processing methodologies were not found, with the exception of $\mathrm{Ru}$ and LE samples. The obtained PUFA/SFA and n-6/n-3 ratio were in accordance to the limits recommended by the World Health Organization (higher than 0.45 and below 4.0, respectively). ${ }^{[23]}$

In this study, the atherogenicity (AI) and thrombogenecity (TI) indexes (Table 6) were evaluated. The AI among the bee pollen extracts ranged between $0.22 \pm 0.04$ and $0.46 \pm 0.03$; while the TI varied between $0.14 \pm 0.01$ and $0.26 \pm 0.03$. In general, higher differences were found for the storage method, explaining 32.4 and $27.6 \%$ of the total variance, respectively, for $\mathrm{Al}$ and $\mathrm{Ti}$. 
Table 3. Profile of fatty acids of the frozen and dried apicultural pollen samples (C4-C14).

\begin{tabular}{|c|c|c|c|c|c|c|c|}
\hline Sample & Code & $\mathrm{C} 4: 0\left(\mathrm{~g} 100^{-1} \mathrm{~g}^{-1}\right)$ & $\mathrm{C} 6: 0\left(\mathrm{~g}_{\left.100^{-1} \mathrm{~g}^{-1}\right)}\right.$ & $\mathrm{C} 8: 0\left(\mathrm{~g}_{\left.100^{-1} \mathrm{~g}^{-1}\right)}\right.$ & $\mathrm{C} 10: 0\left(\mathrm{~g} 100^{-1} \mathrm{~g}^{-1}\right)$ & 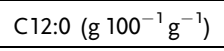 & $\mathrm{C} 14: 0\left({\left.\mathrm{~g} 100^{-1} \mathrm{~g}^{-1}\right)}^{-1}\right.$ \\
\hline \multirow[t]{2}{*}{$\mathrm{Ru}$} & $\mathrm{F}$ & $0.02 \pm 0.02^{\mathrm{a}}$ & $0.03 \pm 0.01^{a}$ & $0.07 \pm 0.01^{\mathrm{a}}$ & $0.01 \pm 0.00^{\mathrm{a}}$ & $0.01 \pm 0.00^{\mathrm{a}}$ & $0.02 \pm 0.01^{\mathrm{a}}$ \\
\hline & D & $0.06 \pm 0.01^{\mathrm{b}}$ & $0.04 \pm 0.01^{a}$ & $0.10 \pm 0.01^{\mathrm{b}}$ & $0.02 \pm 0.01^{\mathrm{a}}$ & $0.02 \pm 0.01^{\mathrm{a}}$ & $0.02 \pm 0.01^{\mathrm{a}}$ \\
\hline \multirow[t]{2}{*}{$\mathrm{Ca}$} & $\mathrm{F}$ & $0.01 \pm 0.02^{\mathrm{a}}$ & $0.05 \pm 0.01^{\mathrm{a}}$ & $0.10 \pm 0.03^{\mathrm{a}}$ & $0.03 \pm 0.01^{\mathrm{a}}$ & $0.01 \pm 0.01^{\mathrm{a}}$ & $0.02 \pm 0.02^{\mathrm{a}}$ \\
\hline & D & $0.04 \pm 0.02^{b}$ & $0.04 \pm 0.01^{\mathrm{a}}$ & $0.10 \pm 0.03^{\mathrm{a}}$ & $0.05 \pm 0.02^{b}$ & $0.03 \pm 0.01^{b}$ & $0.03 \pm 0.02^{\mathrm{a}}$ \\
\hline \multirow[t]{2}{*}{$\mathrm{Ci}$} & $\mathrm{F}$ & $0.03 \pm 0.02^{\mathrm{a}}$ & $0.03 \pm 0.01^{a}$ & $0.08 \pm 0.04^{\mathrm{a}}$ & $0.05 \pm 0.03^{a}$ & $0.03 \pm 0.02^{\mathrm{a}}$ & $0.04 \pm 0.01^{\mathrm{a}}$ \\
\hline & D & $0.04 \pm 0.01^{\mathrm{a}}$ & $0.05 \pm 0.01^{b}$ & $0.11 \pm 0.03^{b}$ & $0.09 \pm 0.01^{b}$ & $0.05 \pm 0.01^{\mathrm{a}}$ & $0.04 \pm 0.03^{a}$ \\
\hline \multirow[t]{2}{*}{ Le } & $\mathrm{F}$ & $0.03 \pm 0.01^{\mathrm{a}}$ & $0.04 \pm 0.02^{\mathrm{a}}$ & $0.05 \pm 0.01^{\mathrm{a}}$ & $0.07 \pm 0.02^{\mathrm{a}}$ & $0.05 \pm 0.03^{\mathrm{a}}$ & $0.06 \pm 0.03^{\mathrm{a}}$ \\
\hline & D & $0.04 \pm 0.01^{\mathrm{a}}$ & $0.07 \pm 0.02^{b}$ & $0.12 \pm 0.02^{b}$ & $0.12 \pm 0.01^{b}$ & $0.08 \pm 0.01^{\mathrm{a}}$ & $0.17 \pm 0.02^{b}$ \\
\hline \multirow[t]{2}{*}{ Cy } & $\mathrm{F}$ & $0.03 \pm 0.02^{\mathrm{a}}$ & $0.03 \pm 0.03^{\mathrm{a}}$ & $0.04 \pm 0.02^{\mathrm{a}}$ & $0.06 \pm 0.04^{\mathrm{a}}$ & $0.04 \pm 0.03^{\mathrm{a}}$ & $0.03 \pm 0.02^{\mathrm{a}}$ \\
\hline & D & $0.08 \pm 0.01^{b}$ & $0.08 \pm 0.01^{b}$ & $0.11 \pm 0.02^{\mathrm{b}}$ & $0.08 \pm 0.03^{\mathrm{a}}$ & $0.10 \pm 0.03^{b}$ & $0.13 \pm 0.05^{\mathrm{b}}$ \\
\hline \multirow[t]{2}{*}{ Ec } & $\mathrm{F}$ & $0.04 \pm 0.01^{\mathrm{a}}$ & $0.04 \pm 0.02^{\mathrm{a}}$ & $0.07 \pm 0.01^{\mathrm{a}}$ & $0.03 \pm 0.02^{\mathrm{a}}$ & $0.03 \pm 0.02^{\mathrm{a}}$ & $0.02 \pm 0.01^{\mathrm{a}}$ \\
\hline & D & $0.08 \pm 0.02^{b}$ & $0.07 \pm 0.02^{\mathrm{a}}$ & $0.11 \pm 0.02^{\mathrm{b}}$ & $0.06 \pm 0.01^{b}$ & $0.07 \pm 0.02^{\mathrm{b}}$ & $0.07 \pm 0.02^{\mathrm{b}}$ \\
\hline \multirow[t]{2}{*}{$\mathrm{Ca}$ and $\mathrm{Ci}$} & $\mathrm{F}$ & $0.05 \pm 0.02^{\mathrm{a}}$ & $0.05 \pm 0.02^{\mathrm{a}}$ & $0.10 \pm 0.02^{\mathrm{a}}$ & $0.07 \pm 0.01^{\mathrm{a}}$ & $0.05 \pm 0.01^{\mathrm{a}}$ & $0.08 \pm 0.01^{\mathrm{a}}$ \\
\hline & D & $0.08 \pm 0.01^{b}$ & $0.07 \pm 0.02^{\mathrm{a}}$ & $0.16 \pm 0.02^{b}$ & $0.10 \pm 0.02^{b}$ & $0.04 \pm 0.01^{a}$ & $0.08 \pm 0.01^{a}$ \\
\hline \multirow[t]{2}{*}{ Eu and $\mathrm{Er}$} & $\mathrm{F}$ & $0.05 \pm 0.01^{\mathrm{a}}$ & $0.05 \pm 0.01^{\mathrm{a}}$ & $0.07 \pm 0.02^{\mathrm{a}}$ & $0.06 \pm 0.03^{\mathrm{a}}$ & $0.07 \pm 0.02^{\mathrm{a}}$ & $0.03 \pm 0.02^{\mathrm{a}}$ \\
\hline & D & $0.07 \pm 0.02^{\mathrm{a}}$ & $0.08 \pm 0.01^{b}$ & $0.11 \pm 0.02^{b}$ & $0.09 \pm 0.01^{b}$ & $0.09 \pm 0.01^{\mathrm{a}}$ & $0.08 \pm 0.01^{b}$ \\
\hline \multirow[t]{2}{*}{$\mathrm{Er}$} & $\mathrm{F}$ & $0.03 \pm 0.02^{\mathrm{a}}$ & $0.04 \pm 0.01^{\mathrm{a}}$ & $0.06 \pm 0.01^{\mathrm{a}}$ & $0.02 \pm 0.01^{a}$ & $0.03 \pm 0.03^{\mathrm{a}}$ & $0.07 \pm 0.02^{\mathrm{a}}$ \\
\hline & D & $0.06 \pm 0.03^{b}$ & $0.08 \pm 0.01^{b}$ & $0.10 \pm 0.04^{b}$ & $0.07 \pm 0.01^{b}$ & $0.06 \pm 0.01^{\mathrm{a}}$ & $0.08 \pm 0.02^{\mathrm{a}}$ \\
\hline
\end{tabular}

Ru, Rubus spp.; Ca, Castaneia sativa; Qu, Quercus spp.; Le, Leontondon spp.; Cy, Cytisus spp.; Ec, Echium spp.; Ci, Cistus spp.; Eu, Eucalyptus spp.; Er, Erica spp. C4:0, butyric acid; C6:0, caproic acid; C8:0, caprylic acid; C10:0, capric acid; C12:0, lauric acid; C14:0, myristoleic acid.

Table 4. Profile of fatty acids of the frozen and dried apicultural pollen samples (C16-C18).

\begin{tabular}{|c|c|c|c|c|c|c|}
\hline Sample & Code & $\mathrm{C} 16: 0\left(\mathrm{~g} 100^{-1} \mathrm{~g}^{-1}\right)$ & 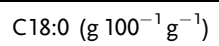 & $\mathrm{C} 18: \ln 9 \mathrm{ct}\left(\mathrm{g} 100^{-1} \mathrm{~g}^{-1}\right)$ & $\mathrm{C} 18: 3 \mathrm{n} 3\left(\mathrm{~g} 100^{-1} \mathrm{~g}^{-1}\right)$ & $\mathrm{C} 18: 2 \mathrm{n} 6 \mathrm{c}\left(\mathrm{g} 100^{-1} \mathrm{~g}^{-1}\right)$ \\
\hline \multirow[t]{2}{*}{ Ru } & $\mathrm{F}$ & $0.50 \pm 0.09^{\mathrm{a}}$ & $0.06 \pm 0.03^{\mathrm{a}}$ & $0.44 \pm 0.06^{\mathrm{a}}$ & $1.71 \pm 0.22^{\mathrm{a}}$ & $0.70 \pm 0.17^{\mathrm{a}}$ \\
\hline & D & $0.86 \pm 0.14^{b}$ & $0.05 \pm 0.01^{\mathrm{a}}$ & $0.51 \pm 0.07^{\mathrm{a}}$ & $1.51 \pm 0.16^{\mathrm{a}}$ & $0.96 \pm 0.02^{\mathrm{b}}$ \\
\hline \multirow[t]{2}{*}{$\mathrm{Ca}$} & $\mathrm{F}$ & $0.52 \pm 0.12^{\mathrm{a}}$ & $0.05 \pm 0.02^{\mathrm{a}}$ & $0.40 \pm 0.08^{\mathrm{a}}$ & $1.32 \pm 0.48^{\mathrm{a}}$ & $0.85 \pm 0.08^{\mathrm{a}}$ \\
\hline & D & $0.68 \pm 0.16^{\mathrm{b}}$ & $0.10 \pm 0.02^{\mathrm{b}}$ & $0.65 \pm 0.09^{\mathrm{b}}$ & $1.67 \pm 0.10^{\mathrm{b}}$ & $0.90 \pm 0.07^{\mathrm{a}}$ \\
\hline \multirow[t]{2}{*}{$\mathrm{Ci}$} & $\mathrm{F}$ & $0.53 \pm 0.17^{\mathrm{a}}$ & $0.09 \pm 0.01^{\mathrm{a}}$ & $0.65 \pm 0.10^{\mathrm{a}}$ & $1.44 \pm 0.22^{\mathrm{a}}$ & $0.85 \pm 0.09^{\mathrm{a}}$ \\
\hline & D & $0.73 \pm 0.05^{\mathrm{a}}$ & $0.08 \pm 0.01^{\mathrm{a}}$ & $0.95 \pm 0.07^{\mathrm{b}}$ & $1.79 \pm 0.16^{\mathrm{a}}$ & $0.99 \pm 0.09^{\mathrm{a}}$ \\
\hline \multirow[t]{2}{*}{ Le } & $\mathrm{F}$ & $0.73 \pm 0.15^{\mathrm{a}}$ & $0.12 \pm 0.04^{\mathrm{a}}$ & $0.93 \pm 0.17^{\mathrm{a}}$ & $1.79 \pm 0.13^{\mathrm{a}}$ & $0.86 \pm 0.05^{\mathrm{a}}$ \\
\hline & D & $1.08 \pm 0.07^{b}$ & $0.13 \pm 0.03^{b}$ & $1.16 \pm 0.14^{\mathrm{a}}$ & $1.90 \pm 0.10^{\mathrm{a}}$ & $1.32 \pm 0.11^{b}$ \\
\hline \multirow[t]{2}{*}{ Cy } & $\mathrm{F}$ & $0.67 \pm 0.16^{\mathrm{a}}$ & $0.06 \pm 0.01^{\mathrm{a}}$ & $0.67 \pm 0.06^{\mathrm{a}}$ & $1.39 \pm 0.15^{\mathrm{a}}$ & $0.79 \pm 0.21^{\mathrm{a}}$ \\
\hline & D & $0.97 \pm 0.12^{\mathrm{a}}$ & $0.11 \pm 0.04^{\mathrm{a}}$ & $1.24 \pm 0.07^{\mathrm{a}}$ & $1.27 \pm 0.24^{\mathrm{a}}$ & $0.93 \pm 0.30^{\mathrm{a}}$ \\
\hline \multirow[t]{2}{*}{ Ec } & $\mathrm{F}$ & $0.51 \pm 0.07^{\mathrm{a}}$ & $0.08 \pm 0.02^{\mathrm{a}}$ & $0.46 \pm 0.18^{\mathrm{a}}$ & $1.74 \pm 0.17^{\mathrm{a}}$ & $0.92 \pm 0.03^{\mathrm{a}}$ \\
\hline & D & $0.96 \pm 0.23^{b}$ & $0.12 \pm 0.01^{\mathrm{b}}$ & $0.84 \pm 0.17^{\mathrm{a}}$ & $2.15 \pm 0.09^{\mathrm{b}}$ & $1.05 \pm 0.14^{\mathrm{a}}$ \\
\hline \multirow[t]{2}{*}{$\mathrm{Ca}$ and $\mathrm{Ci}$} & $\mathrm{F}$ & $0.69 \pm 0.09^{\mathrm{a}}$ & $0.08 \pm 0.01^{\mathrm{a}}$ & $0.62 \pm 0.12^{\mathrm{a}}$ & $1.77 \pm 0.02^{\mathrm{a}}$ & $0.78 \pm 0.17^{\mathrm{a}}$ \\
\hline & D & $0.76 \pm 0.18^{\mathrm{a}}$ & $0.12 \pm 0.02^{\mathrm{b}}$ & $0.98 \pm 0.13^{\mathrm{b}}$ & $2.02 \pm 0.28^{\mathrm{b}}$ & $0.97 \pm 0.06^{\mathrm{a}}$ \\
\hline \multirow[t]{2}{*}{$\mathrm{Eu}$ and $\mathrm{Er}$} & $\mathrm{F}$ & $0.61 \pm 0.06^{\mathrm{a}}$ & $0.06 \pm 0.01^{\mathrm{a}}$ & $0.65 \pm 0.08^{\mathrm{a}}$ & $1.92 \pm 0.12^{\mathrm{a}}$ & $0.95 \pm 0.08^{a}$ \\
\hline & D & $0.98 \pm 0.03^{b}$ & $0.11 \pm 0.02^{\mathrm{b}}$ & $0.91 \pm 0.14^{\mathrm{b}}$ & $1.97 \pm 0.02^{\mathrm{a}}$ & $1.01 \pm 0.20^{\mathrm{a}}$ \\
\hline \multirow[t]{2}{*}{$\mathrm{Er}$} & $\mathrm{F}$ & $0.40 \pm 0.12^{\mathrm{a}}$ & $0.06 \pm 0.01^{\mathrm{a}}$ & $0.36 \pm 0.10^{\mathrm{a}}$ & $1.50 \pm 0.18^{\mathrm{a}}$ & $0.74 \pm 0.20^{\mathrm{a}}$ \\
\hline & D & $0.75 \pm 0.12^{b}$ & $0.10 \pm 0.03^{b}$ & $0.96 \pm 0.04^{b}$ & $1.77 \pm 0.36^{a}$ & $1.02 \pm 0.27^{\mathrm{a}}$ \\
\hline
\end{tabular}

Ru, Rubus spp.; Ca, Castaneia sativa; Qu, Quercus spp.; Le, Leontondon spp.; Cy, Cytisus spp.; Ec, Echium spp.; Ci, Cistus spp.; Eu, Eucalyptus spp.; Er, Erica spp. C16:0, palmitic acid; C18:0, stearic acid; C18:1n9ct, oleic acid; C18:3n3, $\alpha$-linolenic acid; C18:2n6c, linoleic acid methyl ester. 
Table 5. Component variance analysis for the fatty acids content in pollen from different species and two storage methods (variance percentage and significance level).

\begin{tabular}{lcccc}
\hline & Species (S) & Storage method (SM) & S $\times$ SM & Residual \\
\hline TF $\left(\mathrm{g} 100^{-1} \mathrm{~g}^{-1}\right)$ & $20.1 \%^{* * *}$ & $61.9 \%^{* * *}$ & $0.0 \% \mathrm{~ns}$ & $18.0 \%$ \\
SFA & $17.9 \%^{* * *}$ & $67.7 \%^{* * *}$ & $0.0 \% \mathrm{~ns}$ & $14.5 \%$ \\
MUFA (C18:1) & $29.9 \%^{* * *}$ & $50.3 \%^{* * *}$ & $9.0 \%^{*}$ & $10.8 \%$ \\
PUFA & $27.3 \%^{* * *}$ & $27.2 \%^{* * *}$ & $0.0 \% \mathrm{~ns}$ & $45.5 \%$ \\
N.I. & $0.0 \% \mathrm{~ns}$ & $35.3 \%^{* * *}$ & $0.0 \% \mathrm{~ns}$ & $64.7 \%$ \\
C4:0 & $20.5 \%^{* * *}$ & $47.0 \%^{* * *}$ & $0.0 \% \mathrm{~ns}$ & $32.5 \%$ \\
C6:0 & $12.3 \%^{*}$ & $44.0 \%^{* * *}$ & $13.3 \% *$ & $30.4 \%$ \\
C8:0 & $14.5 \%^{* *}$ & $56.2 \%^{* * *}$ & $0.0 \% \mathrm{~ns}$ & $29.3 \%$ \\
C10:0 & $43.6 \%^{* * *}$ & $35.8 \%^{* * *}$ & $0.0 \% \mathrm{~ns}$ & $20.6 \%$ \\
C12:0 & $42.7 \%^{* * *}$ & $27.9 \%^{* * *}$ & $0.0 \% \mathrm{~ns}$ & $29.4 \%$ \\
C14:0 & $32.2 \%^{* * *}$ & $25.0 \%^{* * *}$ & $28.0 \% * *$ & $14.8 \%$ \\
C16:0 & $13.4 \%^{* * *}$ & $61.5 \%^{* * *}$ & $0.0 \% \mathrm{~ns}$ & $25.0 \%$ \\
C18:0 & $19.5 \%^{* *}$ & $33.6 \%^{* * *}$ & $17.1 \%{ }^{*}$ & $29.8 \%$ \\
C18:3n3 & $41.5 \%^{* * *}$ & $12.7 \%^{* *}$ & $0.0 \% \mathrm{~ns}$ & $45.8 \%$ \\
C18:2n6c & $0.0 \% \mathrm{~ns}$ & $42.5 \%^{* * *}$ & $0.0 \% \mathrm{~ns}$ & $57.5 \%$ \\
\hline
\end{tabular}

TF, total fat; SFA, saturated fatty acids; MUFA, monounsaturated fatty acids; PUFA, poly unsaturated fatty acids; N.I., non-identified fatty acids; C4:0, butyric acid; C6:0, caproic acid; C8:0, caprylic acid; C10:0, capric acid; C12:0, lauric acid; C14:0, myristoleic acid; C16:0, palmitic acid; C18:0, stearic acid; C18:3n3, $\alpha$ linolenic acid; C18:2n6c, linoleic acid methyl ester.

${ }^{*}$ Significant $(0.01<p<0.05), \quad{ }^{* *}$ very significant $(0.001<p<0.01), \quad{ }^{* * *}$ highly significant $(p<0.001)$, ns, not significant $(p>0.05)$.

Indeed, significant lower values were obtained on the majority of the frozen pollen samples. Concerning the effect of the species, it explained $16.7 \%$ of the total variance obtained for AI, yet only

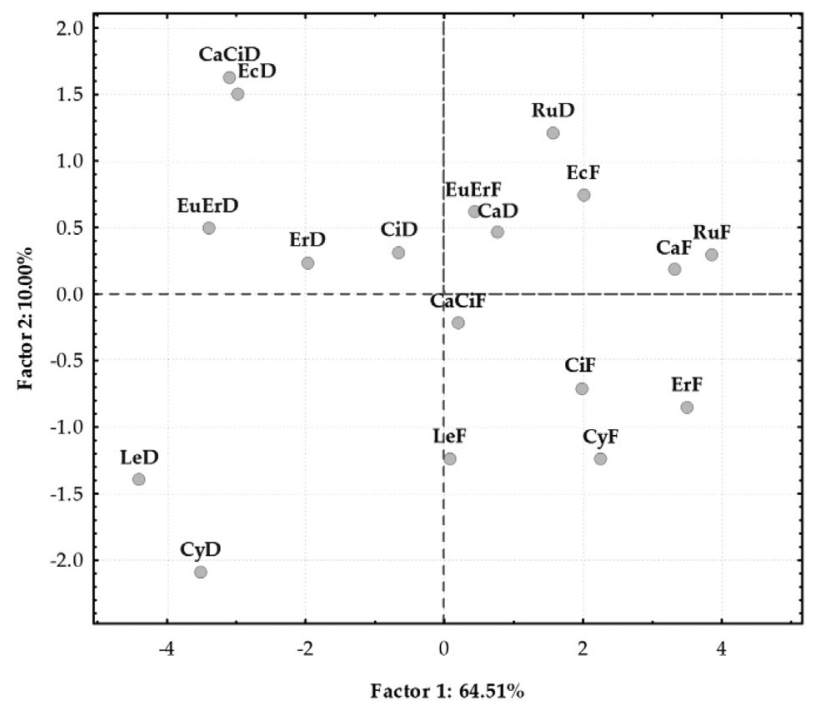

Figure 1. Projection of the pollen samples on the plane of the two main factors resulting from principal component analysis for the individual fatty acid (C4, C6, C8, C10, C12; C14; C16, C18; C18:1, C18:2, C18:3).
$9 \%$ of TI variance. This suggests that the lipid indexes are more influenced by the storage methodology than by the bee pollen type.

The extract Ec and Ru were, for both conservation processes, those that presented the lowest AI and TI indexes, suggesting that they are the most suitable for a healthy diet.

Considering the ANOVA results, presented in the end of Table 6, the factor that most influenced the PUFA/SFA and AI was the storage method that allowed to explain 47.5 and $32.4 \%$ of the total variance, respectively. Once again, the high variability observed (residual: $22.2 \%$ for $\mathrm{Al}$ and $63.4 \%$ for $\mathrm{TI}$ ) may be associated with the natural variability of the bee pollen and another factors that were not evaluated in this study.

\subsection{Vitamin $C, \beta$-Carotene and Lycopene}

In Table 7, it is depicted the amounts of vitamin C, $\beta$-carotene, and lycopene. The vitamin $\mathrm{C}$ content among the pollen extracts ranged between $22.9 \pm 2.04$ and $214.36 \pm 18.43 \mu \mathrm{gg}^{-1}$. The concentration of vitamin $\mathrm{C}$ was higher in the frozen Ca extract $\left(214.36 \pm 18.43 \mu \mathrm{gg}^{-1}\right)$, followed by frozen Le $(143.73 \pm 13.75$ $\left.\mu \mathrm{g} \mathrm{g}^{-1}\right)$. The amount of vitamin $\mathrm{C}$ differed between the preservation processes, for all the extracts.

Regarding the content of $\beta$-carotene, the frozen Er extract presented the highest concentration $\left(84.79 \pm 12.31 \mu \mathrm{gg}^{-1}\right)$, while the lowest value was obtained for the dehydrated $\mathrm{Ru}$ extract $\left(6.42 \pm 0.80 \mu \mathrm{g} \mathrm{g}^{-1}\right)$.

The concentration of lycopene ranged between $1.92 \pm 0.14$ to $59.18 \pm 1.42 \mu \mathrm{gg}^{-1}$, whereas the highest content was found in the frozen sample $\mathrm{Eu}+\mathrm{Er}$.

Table 7 shows the variance analysis for the vitamin $C, \beta$ carotene, and lycopene content in bee pollen from different species and two storage methods. For lycopene the differences between species explain almost all variance observed (88.2\%) moreover the storage method is also highly significant (Table 7). For vitamin $C$ and $\beta$ carotene the effect of species and storage method are highly significant and are quite similar.

\section{Discussion}

According to the literature, the botanical origin of bee pollen is influenced by the harvest region, climate, and vegetation available for bees at the time of harvest. ${ }^{[24]}$

The differences observed in the amount of SFA and MUFA may be related to structural changes induced by thermal processing, among which the un-catalyzed reaction between fats and water dissolved in the fat phase at suitable temperatures ${ }^{[25]}$ but also to the thermic influence on enzymatic activity. In fact, during the drying process bee pollen is heated in an electric oven to $42^{\circ} \mathrm{C}$ which is near the optimum temperature for lipase activation $\left(40^{\circ} \mathrm{C}\right)$, therefore promoting the hydrolysis of fatty acids. ${ }^{[26]}$ The presence of lipase on beehive products, particularly bee pollen, has been reported by some authors ${ }^{[3]}$ and its source is believed to be the salivary glands of the honeybee, especially the labial ones.

Our results are in agreement with the observations of Wanyo et al. ${ }^{[26]}$ and You et al. ${ }^{[27]}$ who studied the fatty acid profiles of rice 
Table 6. Lipid ratios, atherogenicity and thrombogenicity indices of the bee pollen samples.

\begin{tabular}{|c|c|c|c|c|c|}
\hline Sample & Code & $\mathrm{n} 6 / \mathrm{n} 3$ & PUFA/SFA & $\mathrm{Al}$ & $\mathrm{TI}$ \\
\hline \multirow[t]{2}{*}{ Ru } & $\mathrm{F}$ & $0.41 \pm 0.06^{\mathrm{a}}$ & $3.37 \pm 0.54^{\mathrm{a}}$ & $0.22 \pm 0.04^{\mathrm{a}}$ & $0.16 \pm 0.03^{\mathrm{a}}$ \\
\hline & D & $0.64 \pm 0.07^{b}$ & $2.17 \pm 0.45^{\mathrm{b}}$ & $0.33 \pm 0.07^{\mathrm{a}}$ & $0.21 \pm 0.04^{b}$ \\
\hline \multirow[t]{2}{*}{$\mathrm{Ca}$} & $\mathrm{F}$ & $0.69 \pm 0.20^{\mathrm{a}}$ & $2.79 \pm 0.18^{\mathrm{a}}$ & $0.25 \pm 0.04^{\mathrm{a}}$ & $0.14 \pm 0.03^{\mathrm{a}}$ \\
\hline & D & $0.54 \pm 0.02^{\mathrm{a}}$ & $2.42 \pm 0.51^{\mathrm{a}}$ & $0.28 \pm 0.05^{\mathrm{a}}$ & $0.19 \pm 0.04^{\mathrm{a}}$ \\
\hline \multirow[t]{2}{*}{$\mathrm{Ci}$} & $\mathrm{F}$ & $0.59 \pm 0.03^{\mathrm{a}}$ & $2.61 \pm 0.34^{a}$ & $0.27 \pm 0.05^{\mathrm{a}}$ & $0.16 \pm 0.04^{\mathrm{a}}$ \\
\hline & D & $0.55 \pm 0.00^{\mathrm{a}}$ & $2.34 \pm 0.15^{\mathrm{a}}$ & $0.26 \pm 0.01^{\mathrm{a}}$ & $0.18 \pm 0.01^{\mathrm{a}}$ \\
\hline \multirow[t]{2}{*}{ Le } & $\mathrm{F}$ & $0.49 \pm 0.06^{\mathrm{a}}$ & $2.35 \pm 0.46^{b}$ & $0.30 \pm 0.08^{\mathrm{a}}$ & $0.20 \pm 0.04^{\mathrm{a}}$ \\
\hline & D & $0.70 \pm 0.09^{b}$ & $1.80 \pm 0.08^{a}$ & $0.43 \pm 0.02^{b}$ & $0.22 \pm 0.02^{\mathrm{a}}$ \\
\hline \multirow[t]{2}{*}{ Cy } & $\mathrm{F}$ & $0.56 \pm 0.09^{\mathrm{a}}$ & $2.31 \pm 0.26^{b}$ & $0.30 \pm 0.05^{\mathrm{a}}$ & $0.19 \pm 0.04^{\mathrm{a}}$ \\
\hline & D & $0.72 \pm 0.11^{\mathrm{a}}$ & $1.32 \pm 0.12^{\mathrm{a}}$ & $0.46 \pm 0.03^{b}$ & $0.26 \pm 0.03^{b}$ \\
\hline \multirow[t]{2}{*}{ Ec } & $\mathrm{F}$ & $0.53 \pm 0.04^{\mathrm{a}}$ & $3.24 \pm 0.51^{\mathrm{b}}$ & $0.22 \pm 0.03^{\mathrm{a}}$ & $0.14 \pm 0.02^{\mathrm{a}}$ \\
\hline & D & $0.49 \pm 0.05^{\mathrm{a}}$ & $2.12 \pm 0.38^{a}$ & $0.33 \pm 0.07^{\mathrm{a}}$ & $0.23 \pm 0.05^{b}$ \\
\hline \multirow[t]{2}{*}{$\mathrm{Ca}$ and $\mathrm{Ci}$} & $\mathrm{F}$ & $0.44 \pm 0.10^{\mathrm{a}}$ & $2.17 \pm 0.14^{\mathrm{a}}$ & $0.34 \pm 0.02^{\mathrm{a}}$ & $0.22 \pm 0.03^{\mathrm{a}}$ \\
\hline & D & $0.49 \pm 0.07^{\mathrm{a}}$ & $2.15 \pm 0.36^{\mathrm{a}}$ & $0.30 \pm 0.05^{\mathrm{a}}$ & $0.20 \pm 0.05^{\mathrm{a}}$ \\
\hline \multirow[t]{2}{*}{ Eu and $\mathrm{Er}$} & $\mathrm{F}$ & $0.50 \pm 0.07^{\mathrm{a}}$ & $2.89 \pm 0.39^{b}$ & $0.23 \pm 0.03^{\mathrm{a}}$ & $0.15 \pm 0.01^{\mathrm{a}}$ \\
\hline & D & $0.51 \pm 0.10^{\mathrm{a}}$ & $1.86 \pm 0.12^{\mathrm{a}}$ & $0.36 \pm 0.03^{b}$ & $0.24 \pm 0.03^{b}$ \\
\hline \multirow[t]{2}{*}{ Er } & $\mathrm{F}$ & $0.49 \pm 0.09^{\mathrm{a}}$ & $3.21 \pm 0.29^{b}$ & $0.28 \pm 0.02^{\mathrm{a}}$ & $0.14 \pm 0.01^{\mathrm{a}}$ \\
\hline & $\mathrm{D}$ & $0.58 \pm 0.08^{\mathrm{a}}$ & $2.13 \pm 0.31^{\mathrm{a}}$ & $0.31 \pm 0.05^{\mathrm{a}}$ & $0.19 \pm 0.02^{b}$ \\
\hline \multicolumn{2}{|l|}{ Species (S) } & $10.9^{*}$ & $15.1^{* * *}$ & $16.7^{* * *}$ & $9.0^{*}$ \\
\hline \multicolumn{2}{|l|}{ Storage method (SM) } & $8.3^{*}$ & $47.5^{* * *}$ & $32.4^{* * *}$ & $27.6^{* * *}$ \\
\hline \multicolumn{2}{|l|}{$S \times S M$} & $43.2^{* *}$ & $16.7^{*}$ & $28.7^{* *}$ & - \\
\hline \multicolumn{2}{|l|}{ Residual } & 37.6 & 20.7 & 22.2 & 63.4 \\
\hline
\end{tabular}

Ru, Rubus spp.; Ca, Castaneia sativa; Qu, Quercus spp.; Le, Leontondon spp.; Cy, Cytisus spp.; Ec, Echium spp.; Ci, Cistus spp.; Eu, Eucalyptus spp.; Er, Erica spp. ${ }^{*}$ Significant $(0.01<p<0.05),{ }^{* *}$ very significant $(0.001<p<0.01),{ }^{* * *}$ highly significant $(p<0.001)$, ns, not significant $(p>0.05)$.

by-products, and those from Telahigue et al., ${ }^{[28]}$ who analyzed fatty acids from hake and sardinella. These authors also reported that drying caused significant increases in saturated fatty acids (SFA) and MUFA.

The fatty acid profile obtained in this work was similar to that reported in previous studies. ${ }^{[8,10,29-32]}$ However, when studying Romanian pollen, Mărgăoan et al. ${ }^{[6]}$ observed greater diversity: 14 fatty acids were identified. Other studies performed by Kostić et al. ${ }^{[8]}$ reported 20 fatty acid in Serbian bee-pollen. As stated by Saa-Otero et al., ${ }^{[32]}$ these variations are due, partly, to the fact that these compounds may result from the secondary metabolism of plants, which themselves are variable. Apart from the importance of the botanical origin, it has been reported that the lipid profile of bee pollen depends on the geographic origin, ${ }^{[29,30]}$ edaphoclimatic conditions as well as on the apicultural practices. $^{[6]}$

In our study the most abundant fatty acids were palmitic, oleic, and linolenic, what is in agreement with the results plotted by Kostić et al. ${ }^{[8]}$

The most abundant saturated, mono- and poly unsaturated fatty acids were palmitic, oleic, and linolenic acid, respectively.

According to the literature, palmitic acid (C16:0) is considered to be atherogenic whereas stearic acid $(\mathrm{C} 18: 0)$ is thrombogenic. ${ }^{[33]}$ Indeed, lauric (C12:0), myristic (C14:0), palmitic (C16:0), and stearic (C18:0) acids have been reported to inhibit endothelium-dependent and independent vasodilation suggesting that these FA may have a role in atherosclerosismediated endothelium dysfunction and, therefore, be considered risk factors for cardiovascular and metabolic diseases. ${ }^{[34]}$ In this context, American Heart Association (AHA) recommends a decrease in the dietary intake of C16:0, C14:0, and C12:0. Concerning these recommendations, the values obtained in for the fatty acids C4:0, C6:0, C8:0, C10:0, C12:0, C14:0, and C18:0 confirm the importance of the consuming bee pollen for health.

Concerning the variability observed in the pollen samples measured by the residual in ANOVA table, the results are in accordance with reposted also by other authors. ${ }^{[35,36]}$

The AI and TI indexes consider the different effects that each fatty acid has on human health, particularly on the probability of increasing atherosclerosis and/or promoting thrombus formation. As expected, these indexes were low and similar to the values reported for other health-promoting food products.

Vitamin C, $\beta$-carotene, and lycopene are important dietary anti-oxidants. Vitamin $\mathrm{C}$ is a physiological antioxidant with very strong reducing power that is easily regenerated via ubiquitous reductants. Indeed, ascorbic acid plays a key role in the protective mechanism against lipid peroxidation due to its ability to directly eliminate reactive oxygen and nitrogen species (ROS and RNS) 
Table 7. Vitamin C, $\beta$ carotene and lycopene content of the bee pollen samples.

\begin{tabular}{|c|c|c|c|c|}
\hline Sample & Code & Vitamin $C\left(\mathrm{mg} \mathrm{g}^{-1}\right)$ & $\beta$-Carotene $\left(\mathrm{mg} \mathrm{g}^{-1}\right)$ & Lycopene $\left(\mathrm{mg} \mathrm{g}^{-1}\right)$ \\
\hline \multirow[t]{2}{*}{$\mathrm{Ru}$} & $\mathrm{F}$ & $46.9 \pm 3.6^{\mathrm{b}}$ & $16.07 \pm 1.41^{b}$ & $9.40 \pm 0.91^{b}$ \\
\hline & $\mathrm{D}$ & $17.5 \pm 2.6^{\mathrm{a}}$ & $6.42 \pm 0.80^{\mathrm{a}}$ & $5.75 \pm 0.75^{\mathrm{a}}$ \\
\hline \multirow[t]{2}{*}{$\mathrm{Ca}$} & $\mathrm{F}$ & $214.4 \pm 18.4^{\mathrm{b}}$ & $53.81 \pm 4.48^{\mathrm{b}}$ & $49.67 \pm 3.85^{\mathrm{b}}$ \\
\hline & D & $143.7 \pm 13.7^{\mathrm{a}}$ & $26.49 \pm 3.95^{\mathrm{a}}$ & $35.67 \pm 1.23^{\mathrm{a}}$ \\
\hline \multirow[t]{2}{*}{$\mathrm{Ci}$} & $\mathrm{F}$ & $70.3 \pm 3.5^{\mathrm{b}}$ & $21.63 \pm 1.84^{\mathrm{b}}$ & $3.34 \pm 0.80^{\mathrm{a}}$ \\
\hline & D & $29.9 \pm 1.8^{\mathrm{a}}$ & $10.84 \pm 1.49^{\mathrm{a}}$ & $1.92 \pm 0.14^{\mathrm{a}}$ \\
\hline \multirow[t]{2}{*}{ Le } & $\mathrm{F}$ & $148.9 \pm 4.7^{b}$ & $45.29 \pm 1.55^{\mathrm{b}}$ & $25.49 \pm 3.02^{\mathrm{b}}$ \\
\hline & D & $54.5 \pm 6.5^{\mathrm{a}}$ & $16.78 \pm 1.78^{\mathrm{a}}$ & $17.44 \pm 1.16^{\mathrm{a}}$ \\
\hline \multirow[t]{2}{*}{ Cy } & $\mathrm{F}$ & $100.2 \pm 14.6^{b}$ & $20.54 \pm 0.57^{b}$ & $9.3 \pm 1.14^{\mathrm{a}}$ \\
\hline & D & $39.2 \pm 3.2^{\mathrm{a}}$ & $10.03 \pm 1.79^{\mathrm{a}}$ & $7.76 \pm 0.23^{\mathrm{a}}$ \\
\hline \multirow[t]{2}{*}{ Ec } & $\mathrm{F}$ & $99.3 \pm 9.8^{\mathrm{b}}$ & $22.78 \pm 1.34^{\mathrm{b}}$ & $5.40 \pm 0.62^{b}$ \\
\hline & D & $22.9 \pm 2.0^{\mathrm{a}}$ & $13.52 \pm 1.58^{\mathrm{a}}$ & $3.58 \pm 0.39^{\mathrm{a}}$ \\
\hline \multirow[t]{2}{*}{$\mathrm{Ca}$ and $\mathrm{Ci}$} & $\mathrm{F}$ & $54.6 \pm 0.2^{b}$ & $17.88 \pm 1.59^{\mathrm{b}}$ & $7.35 \pm 0.63^{b}$ \\
\hline & D & $22.4 \pm 3.7^{\mathrm{a}}$ & $8.93 \pm 1.44^{\mathrm{a}}$ & $5.55 \pm 0.44^{\mathrm{a}}$ \\
\hline \multirow[t]{2}{*}{ Eu and $\mathrm{Er}$} & $\mathrm{F}$ & $124.7 \pm 6.8^{b}$ & $54.12 \pm 3.42^{b}$ & $59.18 \pm 1.42^{\mathrm{b}}$ \\
\hline & $\mathrm{D}$ & $51.8 \pm 5.6^{\mathrm{a}}$ & $17.9 \pm 0.24^{\mathrm{a}}$ & $42.55 \pm 2.70^{\mathrm{a}}$ \\
\hline \multirow[t]{2}{*}{ Er } & $\mathrm{F}$ & $130.6 \pm 3.9 \mathrm{~b}$ & $84.8 \pm 12.3^{b}$ & $31.82 \pm 0.83^{\mathrm{b}}$ \\
\hline & $\mathrm{D}$ & $78.0 \pm 3.6 \mathrm{a}$ & $16.07 \pm 1.41^{\mathrm{a}}$ & $21.28 \pm 2.98^{\mathrm{a}}$ \\
\hline Species (S) & & $52.2 \%^{* * *}$ & $42.7 \% * * *$ & $88.2 \% \%^{* * *}$ \\
\hline Storage method (SM) & & $41.1 \% * * *$ & $36.7 \% * * *$ & $6.1 \%^{* * *}$ \\
\hline$S \times S M$ & & $5.0 \%^{* * *}$ & $18.7 \% * * *$ & $4.9 \%^{* * *}$ \\
\hline Residual & & $1.7 \%$ & $2.0 \%$ & $0.8 \%$ \\
\hline
\end{tabular}

Ru, Rubus spp.; Ca, Castaneia sativa; Qu, Quercus spp.; Le, Leontondon spp.; Cy, Cytisus spp.; Ec, Echium spp.; Ci, Cistus spp.; Eu, Eucalyptus spp.; Er, Erica spp. ${ }^{*}$ Significant $(0.01<p<0.05),{ }^{* *}$ very significant $(0.001<p<0.01),{ }^{* * *}$ highly significant $(p<0.001)$, ns, not significant $(p>0.05)$.

and to regenerate tocopherol species. ${ }^{[37]}$ On the other hand, carotenoids are important precursors of vitamin $\mathrm{A}$ and are known to be very efficient quenchers of oxygen radical and potent scavengers of other reactive oxygen species. ${ }^{[37]}$

The results obtained in the present study for frozen and dehydrated Portuguese bee pollen samples were consistent to those reported by De Melo and Almeida Muradian ${ }^{[12]}$ who studied Brazilian fresh bee pollen (3.77-99.27 $\mu \mathrm{g} \mathrm{g}^{-1}$ ). However, for this parameter Mărgăoan et al. ${ }^{[6]}$ obtained lower values (from trace to $13.2 \mu \mathrm{g} \mathrm{g}^{-1}$ ). These variations can be attributed to the conservation process ${ }^{[12]}$ to the botanical origin of the samples, as well as the climatic conditions. ${ }^{[6]}$

Bee pollen has also been reported to be rich in organic carotenoid, pigments, among which $\beta$-carotene, lycopene and zeaxanthin. These compounds stand out for having antioxidant properties and for improving the immune response. ${ }^{[38]}$ Concerning the lycopene concentration, it was observed differences between the two conservation processes, for all the samples under study.

\section{Conclusions}

The preservation method influenced the amount of SFA and MUFA of bee pollen samples but not the PUFAs concentration, presenting the dried samples a superior fatty-acids' concentration. Even though the storage method appeared to be a highly significant factor for explaining the differences between samples, the botanical origin also influenced the fatty-acids content.

Based on the obtained lipid indexes, it may be inferred that bee pollen is a dietary low risk factor and may even be introduced in foods and beverages to reduce the risks of atherosclerosis and thrombogenesis.

Regarding lycopene, vitamin $\mathrm{C}$, and $\beta$-carotene, the effect of species and storage method were highly significant, presenting frozen bee pollen stored a significantly higher concentration of these important dietary anti-oxidants.

From the plotted results it is possible to infer that it is better to consume bee pollen frozen at $-20^{\circ} \mathrm{C}$ in comparison to that dried in an electric oven.

\section{Abbreviations}

$\mathrm{Al}$, atherogenic; $\mathrm{Ca}$, Castanea sativa; $\mathrm{Ca}$ and $\mathrm{Ci}$, Castanea sativa and Cistus spp.; Cy, Cytisus spp.; EC, Echium spp.; Er, Erica spp.; Eu and Er, Eucalyptus spp. and Erica spp.; L2, Leontondon spp.; MUFA, mono-unsaturated fatty 
acids; PUFA, poly-unsaturated fatty acids; Qu, Quercus spp.; Ru, Rubus spp.; TI, thrombogenic.

\section{Acknowledgments}

Centro de Estudos Florestais is a research unit funded by FCT (UID/AGR/ UI0239/2013). The author L. M. Estevinho wishes to thank to the strategic programme UID/BIA/04050/2013 (POCI-01-0145-FEDER-007569) funded by national funds through the Fundação para a Ciência e a Tecnologia (FCT, Portugal) and by the European Regional Development Fund (ERDF) through the COMPETE2020-Programa Operacional Competitividade e Internacionalização (POCI).

\section{Conflict of Interest}

The authors declare no conflict of interest.

\section{Keywords}

antioxidants, bee pollen, lipid profile, nutritional characteristics, storage conditions

Received: September 12, 2018 Revised: October 29, 2018 Published online:

[1] M. G. Campos, L. Olena, O. Anjos, in Chemistry, Biology and Potential Applications of Honeybee Plant-Derived Products (Eds: S. M. Cardoso, A. M. S. Silva), Bentham Science Publishers, United Arab Emirates 2016, Ch. 45

[2] M. G. Campos, S. Bogdanov, L. B. Almeida-Muradian, T. Szczesna, Y. Mancebo, C. Frigerio, F. Ferreira, J. Apic. Res. 2008, 47, 154.

[3] K. Komosinska-Vassev, P. Olczyk, J. Kaźmierczak, L. Mencner, K. Olczyk, J. Evid. Based Complementary Altern. Med. 2015, 2015, 6.

[4] A. Pascoal, S. Rodrigues, A. Teixeira, X. Feás, L. M. Estevinho, Food Chem. Toxicol. 2014, 63, 233.

[5] T. Szczesna, H. Rybak-Chmielewska, W. Chmielewski, J. Appl. Sci. 2002, 46, 107.

[6] R. Mărgăoan, L. A. Mărghitaş, D. S. Dezmirean, F. V. Dulf, A. Bunea S. A. Socaci, O. Bobiş, J. Agric. Food Chem. 2014, 62, 6306.

[7] S. Ghosh, C. Jung, J. Asia Pac. Entomol. 2017, 20, 245.

[8] A. Ž. Kostić, M. B. Pešić, D. Trbović, R. P. Aleksandra, M. Dramićanin, D. M. Milojković-Opsenica, L. Ž. Tešić, J. Apic. Res. 2017, 56, 533

[9] D. Domínguez-Valhondo, D. Bohoyo Gil, M. T. Hernández, D. GonzálezGómez, Int. J. Food Sci. Technol. 2011, 47, 429.

[10] L. M. Estevinho, S. Rodrigues, A. P. Pereira, X. Feás, Int. J. Food Sci. Technol. 2012, 47, 429.

[11] L. B. Almeida-Muradian, L. C. Pamplona, S. Coimbra, O. M. Barth, J. Food Compost. Anal. 2005, 18, 105.
[12] I. L. P. De Melo, L. B. Almeida-Muradian, Quím. Nova. 2010, 33, 514

[13] A. Rzepecka-Stojko, B. Pilawa, P. Ramos, J. Stojko, J. Appl. Sci. 2012, $56,23$.

[14] S. Collin, T. Vanhavre, E. Bodart, A. Bouseta, J. Agric. Food Chem. 1995, 43, 444.

[15] A. Z. Kostić, M. B. S. P. Barac, D. M. Stanojevic, D. Milojkovic Opsenica, Z. L. Tesic, B. Sikoparija, P. Radisic, M. Prentovic, M. B. Pesic, Food Sci. Technol. 2015, 62, 301.

[16] X. Feás, M. P. Vázquez-Tato, L. Estevinho, J. Á. Seijas, A. Iglesias, Molecules 2012, 17, 8359

[17] M. S. Bárbara, C. S. Machado, G. D. S. Sodré, L. G. Dias, L. M. Estevinho, C. A. L. De Carvalho, Molecules 2015, 20, 12525.

[18] M. Ghaeni, K. N. Ghahfarokhi, L. Zaheri, J. Marine Sci.: Res Dev. 2013, 3, 1 .

[19] G. Montenegro, R. Pizarro, E. Mejías, S. Rodríguez, Phyton 2013 82,7

[20] A. M. Adalid, S. Roselló, F. Nuez, J. Food Compost. Anal. 2010, 23, 613.

[21] AOAC. Association of Official Analytical Chemists; Official Methods of Analysis of AOAC International, 16th ed., AOAC International, Arlington 1995, Ch. 45.

[22] K. C. L. S. Oliveira, M. Moriya, R. A. B. Azedo, L. B. AlmeidaMuradian, Quím. Nova. 2009, 32, 1099.

[23] World Health Organization: Diet, nutrition and the prevention of chronic diseases: report of a joint WHO/FAO expert consultation WHO technical report, 2003, series no. 916, Geneva, Switzerland.

[24] C. F. Da Luz, B. Junior, L. Gabriel, R. L. Fonseca, P. R. D. Sousa, An. Acad. Bras. Ciênc. 2010, 82, 293.

[25] J. Salimon, B. M. Abdullah, N. Salih, Chem. Cent. J. 2011, 5, 1.

[26] P. Wanyo, N. Meeso, S. Siriamornpun, Food Chem. 2014, 157, 457.

[27] J. You, F. Zhu, W. Zhao, X. Zhao, Y. Suo, S. Liu, Eur. J. Lipid. Sci. Technol. 2007, 109, 225.

[28] K. Telahigue, T. Hajji, I. Rabeh, M. E. Cafsi, Afr. J. Biochem. Res. 2013 7, 158.

[29] C. Gardana, C. Del Bo', M. C. Quicazán, A. R. Corrrea, P. Simonetti, J. Food. Compost. Anal. 2018, 73, 29.

[30] G. Conte, G. Benelli, A. Serra, F. Signorini, M. Bientinesi, C. Nicolella, M. Mele, A. Canale, J. Food. Compost. Anal. 2017, $55,12$.

[31] V. A. Isidorov, A. G. Isidorova, L. Sczczepaniak, U. Czyżewska, Food Chem. 2009, 115, 1056.

[32] M. P. Saa-Otero, E. Diaz-Losada, E. Fernandez-Gomez, Grana 2002 39, 175 .

[33] Y. A. Attia, M. A. Al-Harthi, M. A. Korish, M. M. Shiboob, Lipids Health Dis. 2015, 14, 1.

[34] R. H. C. Lee, C. S. Wilkins, A. C. Silva, S. E. Valido, C. Y. C. W. Wu, H. W. Lin, in Fatty Acids in Vascular Health (Ed: L. F. Porto), Nova Science Publishers Pub Inc., Hauppauge, New York 2014, Ch. 1.

[35] P. Gonçalves, M. L. Estevinho, A. P. Pereira, J. M. C. Sousa, O. Anjos, Food Chem. 2018, 267, 36.

[36] O. Anjos, A. J. A. Santos, T. Dias, L. M. Estevinho, J. Apic. Res. 2017 $56,210$.

[37] J. Fiedor, K. Burda, Nutrients 2014, 6, 466.

[38] B. Pinto, F. Caciagli, E. Riccio, D. Reali, A. Šarić, T. Balog, S. Likić, R. Scarpato, Eur. J. Med. Chem. 2011, 45, 4122. 